\title{
Cognitive Behavioral Therapy in Breast Cancer Patients - a Feasibility Study of an 8 Week Intervention for Tumor Associated Fatigue Treatment
}

\author{
Christian Eichler ${ }^{1,2 *}$, Multhaupt Pia $^{2}$,Multhaupt Sibylle ${ }^{2}$,Axel Sauerwald ${ }^{3}$, Wolff, \\ Friedrich $^{1,4}$, Mathias Warm ${ }^{2,4}$
}

\begin{abstract}
Background: Tumor associated fatigue (TAF) or cancer related fatigue (CRF) is not a new concept. Nonetheless, no real headway has been made in the quantitative analysis of its successful treatment via cognitive behavioral therapy. Since 20 to 30\% of all breast cancer patients suffer from anxiety and/or depression within the first year of their diagnosis, this issue needs to be addressed and a standard treatment protocol has to be developed. This study focused on developing a simple, reproducible and short (8 weeks) protocol for the cognitive behavioral therapy support of tumor associated fatigue patients. Materials and Methods: Between the year 2011 and 2012, 23 breast cancer patients fulfilled the diagnosis TAF requirements and were introduced into this study. Our method focused on a psycho-oncological support group using a predetermined, highly structured and reproducible, cognitive behavioral therapy treatment manual. Eight weekly, 90 minute sessions were conducted and patients were evaluated before and after this eight session block. Tumor fatigue specific questionnaires such as the multidimensional fatigue inventory (MFI) as well as the hospital anxiety and depression scale (HADS) were used in order to quantitatively evaluate patient TAF. Results: Of the 23 patients enrolled in the study, only 7 patients fulfilled the TAF diagnostic criteria after the psycho-oncological group treatment. This represents a $70 \%$ reduction in diagnosable tumor associated fatigue. The HADS analysis showed a $33 \%$ reduction in patient anxiety as well as a $\mathbf{5 7 \%}$ reduction in patient depression levels. The MFI scores showed a significant reduction in 4 of the 5 evaluate categories. With the exception of the "mental fatigue" MFI category all results were statistically significant. Conclusions: This study showed that a highly structured, cognitive behavioral therapy group intervention will produce significant improvements in breast cancer patient tumor associated fatigue levels after only 8 weeks.
\end{abstract}

Keywords: Breast cancer - cognitive behavioral therapy - tumor associated fatigue - cancer related fatigue

Asian Pac J Cancer Prev, 16 (3), 1063-1067

\section{Introduction}

Tumor associated fatigue (TAF), also known as cancer related fatigue (CRF), emerges to be a largely neglected factor in the treatment of breast cancer patients. While a plethora of diagnostic developments and treatment options are under continuous evaluation, the main focus remains on the somatic aspects of this disease, i.e. meeting the oncological challenge by medication, chemotherapy, radiation treatment, surgical intervention and standard clinical care (Schumacher, 2003). Largely due to this aggressive somatic treatment of breast cancer, tumor associated fatigue is often diagnosed too late in breast cancer therapy (Montazeri et al., 2001; Fafouti et al., 2010; Schleife et al., 2012; Kovacs et al., 2013; Abu-Helalah et al., 2014). Nonetheless an ever-growing body of literature is becoming available for breast cancer patients suffering from TAF/ CRF.

Although psycho-oncological treatment plays an ever bigger role in modern breast cancer treatment, the subgroup suffering from tumor associated fatigue syndrome is a more recent area of investigation (Larkey et al., 2014; Schmidt et al., 2014). TAF is ambiguously defined and includes patients presenting with avolition and lethargy, as well as a plethora of nonspecific symptoms (Mitchell, 2010; Li et al., 2014). The national comprehensive cancer network (NCNN) recommends two general approaches: medication based and nonmedication based therapy (Montazeri et al., 2001). While the differentiation between a cancer associated depression and a tumor associated fatigue is fluent, the prior should be treated using pharmaceuticals. The latter on the other

${ }^{1}$ Department of Gynecology and Obstetrics, Holweide Hospital, ${ }^{2}$ Brustzentrum, Krankenhaus Holweide, Department of Gynecology and Obstetrics, ${ }^{4}$ University of Cologne, Cologne, ${ }^{3}$ Hospital Düren GmbH, Düren, Germany *For correspondence: ceichler@gmail. com,eichlerc@kliniken-koeln.de 
hand, may be treated by a non-medication based therapy which includes an increase in physical activity, stress- and problem management training, and psycho-oncological therapy/intervention. While it is difficult to determine whether patients suffers from a depression or a TAF, due to the lack of a quick diagnostic tool, it is paramount to determine which is the main source of patient discomfort (Bjorneklett et al., 2012) as the therapy may vary depending on the diagnosis.

20 to $30 \%$ of all breast cancer patients suffer from anxiety and/ or depression within the first year of their diagnosis. The depression generally subsides after approximately one year. Fear and anxiety of a possible recurrence however remain. Several studies showed that both depression and anxiety contribute to and are related to cancer associated fatigue. (Vodermaier and Millman, 2011; Cosco et al., 2012). Differentiating fatigue and depression is difficult to the fact that both are associated with lethargy, tiredness and exhaustion. In order to distinguish the two, a two-question test was established (Roth et al., 2010).

These questions are: $i$ ) Within the last month, did you feel an increasing sadness, hopelessness, and/or gloominess? ii) Within the last month, did you experience less enthusiasm and joy within your daily activities?

If both of these questions are answered with yes, the diagnosis of a depression is more likely. If only one question is answered with "yes" a TAF is possible. This preliminary TAF diagnosis may then be supported by world health organization (WHO) criteria catalogue, determining the severity of a clinically relevant cancer/ tumor associated fatigue. The differentiation between the two is important due to the fact that a fatigue treated with antidepressants does not show any improvement while patients still experience all the side effects of the medication (Minton et al., 2008). The role of a pharmaceutical intervention in TAF patients is currently unclear. While psycho-stimulating substances are being evaluated in studies, no fundamental breakthrough has been made in this area. The main focus of treatment thus lies on increased physical activity and individual psychooncological support and nutrition improvement. All three have shown to lead to a significant improvement in anxiety and depression symptoms in the tumor associated fatigue patients group (Campos et al., 2011; Chan, 2011; Fitch, 2012; Finnegan-John et al., 2013).

The focus of this paper is therefore: $i$ ) Is it possible to positively influence anxiety and depression components of TAF breast cancer patients by, cognitive behavioral therapy group intervention with an 8 week intervention? ii) Is it possible to develop a practical, simple and clearly structured protocol for a psycho-oncological intervention in TAF patients?

\section{Materials and Methods}

Between 2011 and 2012, 24 patients were recruited into the study. The average age was 45 years, one patient discontinued the study due to personal reasons. The remaining 23 patients were either currently in adjuvant breast cancer therapy or had recently finished therapy. The longest time interval between the last oncological therapy session and the beginning of the psycho-oncological intervention was 24 months; anti-hormonal therapy excluded. Tumor associated fatigue was diagnosed using the criteria catalog for a tumor associated fatigue syndrome. The world health organization (WHO) suggested a criteria catalogue which allows for the evaluation of the severity of a clinically relevant cancer associated fatigue. These criteria are also used by the fatigue coalition USA (Curt et al., 2000; Cella et al., 2001) and consist of 11 patient evaluated questions as well as three physician evaluated categories. If six of these fourteen criteria are met, the patient presents with a clinically relevant tumor associated fatigue. Naturally, all 23 of the patients fulfilled this requirement before the first therapy session. Quantitative evaluation of patient TAF status was performed before and after the group sessions were completed. Evaluation tools were the HADS and MFI questionnaire. A study timetable is provided in Figure 1.

\section{Hospital Anxiety and Depression Scale (HADS)}

The HADS is a self-evaluation tool, quantitatively analyzing patient anxiety and depression symptoms. It was administered to patients before and after 8 therapy sessions. Both, anxiety and depression symptoms are scored separately. Each of the seven questions is answered on a scale from 0 to 3 , yielding a total of 21 possible points. 0-7 points is regarded as normal while 8-10 points is a moderate, $11-14$ points a serious and $15-21$ points a very serious symptom constellation (Zigmond and Snaith, 1983).

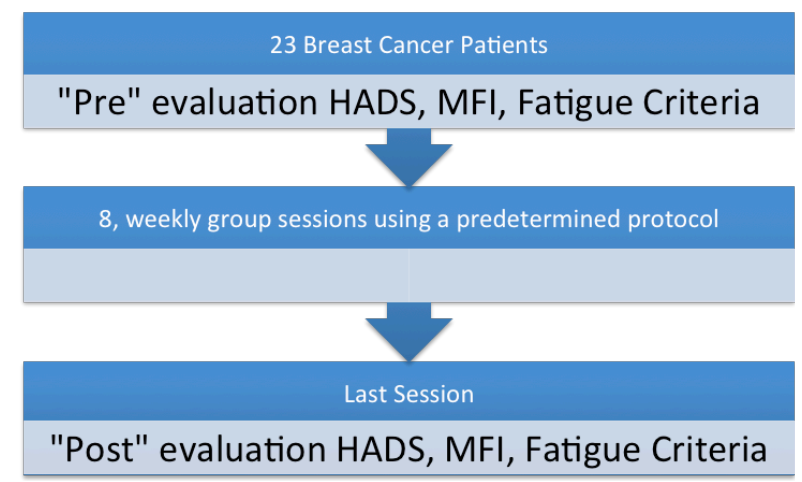

Figure 1. Schematic Representation of the Study Timetable

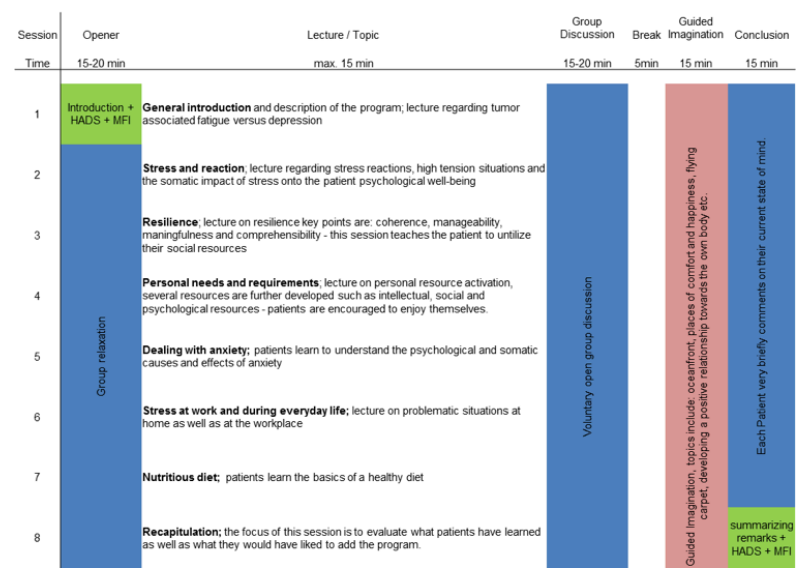

Figure 2. Schematic Representation of Group Sessions 


\section{Multidimensional Fatigue Inventory (MFI)}

The MFI is a 20-item quantitative, self-reported questionnaire designed to evaluate TAF. The following dimensions are evaluated: general fatigue, physical fatigue, mental fatigue, reduced motivation and reduced activity (Smets et al., 1995). Evaluating these questions in a manner that questions directed towards a negative answer were scored on a positive scale when answered with a low numerical value, allowed for a simple compilation of the cumulative data. In order to evaluate the degree of fatigue, an absolute numerical value was assigned to all answers. For example: strong fatigue (5), little fatigue (1). The MFI therefore allows a quantitative correlation of patient fatigue before and after our intervention.

Group Intervention: The basis of this psychooncological intervention is the improvement of patient quality of life. Pre-defined patient goals are: $i$ )Improvement in self-management: patients learn to actively control fatigue symptoms ii) Development of realistic goals iii) Discover and use of available resources $i v$ ) Information on healthy nutrition $v$ ) Learning to deal with tumor associated stress

Each group performed a total of eight sessions, 90 minutes each, once a week over an 8 week period. Each session was led by an experienced psycho-oncologist and was highly structured based on a predetermined manual.

Each session begins with a 10 to 15 minute relaxation exercise, during which self-awareness and relaxation techniques were addressed and practiced. This portion was followed by an educative session regarding several different topics. This lecture was no longer than 15 minutes. A 15 minute open discussion focusing on the subject of each session followed. Before ending each session, a 5 minutes break was introduced giving each patient the option to focus their thoughts on this particular subject. Closing every meeting, each patient was asked to, very briefly, summarize their feelings regarding this topic in two sentences or less. This highly structured lesson scheme was applied to every one of the eight sessions, allowing each patient to prepare themselves for this type of intervention. Figure 2 shows an overview of the group manual.

\section{Statistics}

Statistical analysis was performed using the VassarStats ${ }^{\circledR}$ (Vassar College, Poughkeepsie, NY, USA) statistics program. Pearson's chi-squared tests and t-tests were used in order to evaluate significances when appropriate. A p-value $<0.05$ was considered significant.

\section{Informed Consent}

Written informed consent was obtained from all patients for publication of this manuscript. A copy of the written consent is available for review by the Editorin-Chief of this journal. This study was conducted in accordance with institutional review board standard operating procedures.

\section{Results}

Before beginning therapy, 23 patients fulfilled six or more diagnosis criteria for tumor associated fatigue, thus qualifying them for this trial. As mentioned above, 11 patient self-scored categories were analyzed. The analysis of the diagnostic criteria showed that only 7 of the 23 initial patients would qualify for this trial after 8 therapy sessions, meaning that only seven patients met the criteria for tumor associated fatigue after intervention. This $70 \%$ reduction is statistically significant $\mathrm{p}<0.05$.

MFI: The evaluation of the MFI questionnaire may be divided into five subgroups. These are: general fatigue, physical fatigue, reduced motivation, reduced activity and mental fatigue. Table 1 shows the MFI scores for all five categories before and after treatment. The improvement is significant in all categories after 8 sessions, with the exception of the mental fatigue category. The general fatigue category initially scored with an average value of 16 before intervention, but could be reduced to a value of 13 afterwards. Physical fatigue scores were reduced from 15 to 12 , reduced motivation was decreased from 11 to 9 and reduced activity was scored with 15 before and 12 after therapy. These average scores all differed significantly. The mental fatigue category score was reduced from 14 to 12 , though significance was not established here. A p-value of 0.146 allows for carefully assuming of a trend towards improvement.

HADS: This questionnaire evaluated both an anxiety and depression aspect. The results pertaining to the anxiety aspect showed 18 patients to have a pathological score before the first intervention (i.e. a score above 7). A significant reduction could be shown to 12 patients after 8 sessions. This represents a 33\% reduction in pathological anxiety scores. The average HADS score for anxiety was 12 before and 8 after intervention. These results are statistically significant. Average scores are shown in Table 2.

Prior to the first session, 14 of the 23 patients showed pathological results on the depression scale

Table 1. Multidimensional Fatigue Inventory (MFI) Scores for 5 Categories before and After Psycho Oncological Intervention

\begin{tabular}{lcrr}
\hline & $\begin{array}{c}\text { Pre- } \\
\text { intervention } \\
\mathrm{A}( \pm \text { SD })\end{array}$ & $\begin{array}{c}\text { Post- } \\
\text { intervention }\end{array}$ & $\mathrm{A}( \pm$ SD $)$ \\
\hline General fatigue & $16( \pm 3)$ & $13( \pm 3)$ & $\mathrm{p}$ \\
Physical fatigue & $15( \pm 3)$ & $12( \pm 3)$ & 0.0003 \\
Reduced motivation & $11( \pm 3)$ & $9( \pm 3)$ & 0.0032 \\
Reduced activity & $15( \pm 3)$ & $12( \pm 3)$ & 0.0245 \\
Mental fatique & $14( \pm 4)$ & $12( \pm 4)$ & 0.1460 \\
\hline *A=Average; SD=standard deviation & &
\end{tabular}

Table 2. Depression and Anxiety (HADS) Scores before and After Psych-Oncological Intervention. 0-7 Points= Normal, 8-10 Points=Moderate, 11-14 Points=serious, 15-21 Points=Very Serious Symptom Constellation

\begin{tabular}{lccc}
\hline & $\begin{array}{c}\text { Pre- } \\
\text { intervention } \\
\mathrm{A}( \pm \mathrm{SD})\end{array}$ & $\begin{array}{c}\text { Post- } \\
\text { intervention } \\
\mathrm{A}( \pm \mathrm{SD})\end{array}$ & $\mathrm{p}$ \\
\hline Anxiety & $12( \pm 5)$ & $8( \pm 3)$ & 0.0272 \\
Depression & $9( \pm 5)$ & $5( \pm 4)$ & 0.0016 \\
\hline *A=average; $\mathrm{SD}=$ standard deviation & &
\end{tabular}


(i.e. a score above 7). Four of those patients showed a severe depression and two of them showed a very severe depression. After 8 sessions, only 6 patients retained a score representing a depression. The average score shown in Table 2 could be reduced from 9 to 5 . More importantly however, the initial 14 patients scoring pathologically on the depression scale could be reduced to 6 patients after intervention. This represents a reduction by $57 \%$ in the depression aspects of the HADS evaluation. These results are statistically significant.

\section{Discussion}

Tumor associated fatigue or cancer related fatigue is a problematic and common occurrence in cancer patients. At least 20 to $30 \%$ of all breast cancer patients report fatigue symptoms. These symptoms include tiredness, depressive episodes, weariness and exhaustion and may result in severe somatic consequences. TAF diagnostic criteria were established in 1998 by the fatigue coalition, but unfortunately tumor associated fatigue is not yet classified within the ICD 10 system (Curt et al., 2000; Cella et al., 2001). This may be one of the reasons why tumor associated fatigue is often undiagnosed and untreated. Several studies investigated the effect of non-pharmacological therapy options onto tumor associated fatigue (Kangas et al., 2008). These included increase physical activity, relaxation techniques, psychotherapeutic intervention, nutrition counseling as well as counseled interaction with other patients. Especially data presented at the San Antonio Breast Cancer Symposium 2013 strongly supports physical activity as a positive prognostic factor (Irwin, 2013) in breast cancer patients. A recent meta-analysis investigated the effect of behavioral psychological measures onto fatigue symptoms and was able to also show a positive effect (Duijts et al., 2011). Our study revisited this topic by giving 23 patients the option to undergo a highly structured, cognitive behavioral therapy regimen consisting of eight sessions, 90 minutes each. This is the shortest time period of cognitive behavioral therapy reported in literature to this date (Bjorneklett et al., 2012; Finnegan-John et al., 2013). All of it can be performed as an outpatient procedure. During these sessions, relaxation techniques as well as coping and resource management techniques were taught, practiced and developed in a group setting. The base assumption was that through these techniques, patients will experience a decrease in anxiety and depression symptoms resulting in a patient collective no longer fulfilling the diagnosis criteria for tumor associated fatigue. Thus, a manual was developed, allowing a very structured and reproducible approach to this type of psycho-oncological intervention. Difficult as it may be to quantify results of a psycho-oncological group intervention, two well-established and validated scoring systems were used in order to paint a before and after picture of the patients state of mind. The hospital anxiety and depression scale as well as multidimensional fatigue inventory were utilized to quantify the levels of fatigue, depression and anxiety before and after eight therapy sessions. Initially, 23 patients qualified for this trial by the use of the fatigue coalition diagnosis questionnaire. After completing 8 group sessions only 7 patients retained a pathological TAF diagnosis score. This is a reduction of approximately $70 \%$ and by itself shows the benefit of this program. The quantitative analysis of the MFI scoring system showed a significant reduction in four of five investigated parameters. An improvement in the mental fatigue category could only be shown as a trend. Furthermore, a statistically significant decrease in the average anxiety and average depression score could be shown using the HADS questionnaire. This analysis showed a $33 \%$ reduction in anxiety and $57 \%$ reduction and depression symptoms. It may therefore be said that this type of structured, reproducible, behavioral therapy based psycho-oncological intervention proved useful and adequate in all evaluated dimensions.

While common sense may dictate that teaching relaxation-, coping- and resource management skills, as well as allowing the patient to interact with other patients may be beneficial in the treatment of tumor associated fatigue, this study, although small in patient number, quantitatively proves that this type of intervention should be part of any cancer treatment. Due to the small sample size this work may be prone to over interpretation and larger trials must follow. An interesting question is whether or not additional intervention, i.e. additional sessions would allow for further improvement of these results. While one therapy block showed a significant decrease in anxiety, depression and fatigue symptoms, to a point where $70 \%$ fewer patients would no longer qualify for a TAF diagnosis, it would be certainly be interesting to determine if further intervention produces additional benefit. Based on our results and the results of current meta-analyses regarding physical activity plus psycho-oncological intervention, we currently employ a program which extends these interventions to three sessions coupled with extensive physical therapy.

In conclusion, this feasibility study showed that, although evaluating a small collective, a highly structured, cognitive behavioral therapy based psycho-oncological intervention will produce significant improvement in patient well-being. This may be achieved in a short period of 8 weeks. A 70\% reduction in overall TAF diagnoses, as well as a $33 \%$ reduction in anxiety and a $57 \%$ reduction and depression symptoms could be produced. This leads to a strong recommendation of this study group to include this type of psycho-oncological intervention into all postoperative breast cancer care.

\section{References}

Abu-Helalah M, Al-Hanaqta M, Alshraideh H, et al (2014). Quality of life and psychological well-being of breast cancer survivors in Jordan. Asian Pac J Cancer Prev, 15, 5927-36.

Bjorneklett HG, Lindemalm C, Rosenblad A, et al (2012). A randomised controlled trial of support group intervention after breast cancer treatment: results on anxiety and depression. Acta Oncol, 51, 198-207.

Campos MP, Hassan BJ, Riechelmann R, et al (2011). Cancerrelated fatigue: a review. Rev Assoc Med Bras, 57, 211-9.

Cella D, Davis K, Breitbart W, et al (2001). Cancer-related fatigue: prevalence of proposed diagnostic criteria in a United States sample of cancer survivors. J Clin Oncol, 

19, 3385-91.

Chan R (2011). Cochrane review summary for cancer nursing: drug therapy for the management of cancer-related fatigue. Cancer Nurs, 34, 250-1.

Cosco TD, Doyle F, Ward M, et al (2012). Latent structure of the Hospital Anxiety And Depression Scale: a 10-year systematic review. J Psychosom Res, 72, 180-4.

Curt GA, Breitbart W, Cella D, et al (2000). Impact of cancerrelated fatigue on the lives of patients: new findings from the Fatigue Coalition. Oncologist, 5, 353-60.

Duijts SF, Faber MM, Oldenburg HS, et al (2011). Effectiveness of behavioral techniques and physical exercise on psychosocial functioning and health-related quality of life in breast cancer patients and survivors--a meta-analysis. Psychooncology, 20, 115-26.

Fafouti M, Paparrigopoulos T, Zervas Y, et al (2010). Depression, anxiety and general psychopathology in breast cancer patients: a cross-sectional control study. In Vivo, 24, 803-10.

Finnegan-John J, Molassiotis A, Richardson A, et al (2013). A systematic review of complementary and alternative medicine interventions for the management of cancer-related fatigue. Integr Cancer Ther, 12, 276-90.

Fitch MI (2012). Systematic review and meta-analysis of the correlates of cancer-related fatigue. Evid Based Nurs, 15, 108-9.

Irwin ML CB, Gross C, Ercolano E, et al (2013). Randomized trial of exercise vs. usual care on aromatase inhibitorassociated arthralgias in women with breast cancer: The hormones and physical exercise (HOPE) study San Antonio Breast Cancer Symposium 2013.

Kangas M, Bovbjerg DH, Montgomery GH (2008). Cancerrelated fatigue: a systematic and meta-analytic review of non-pharmacological therapies for cancer patients. Psychol Bull, 134, 700-41.

Kovacs Z, Szabo C, Fulop E (2013). The therapy helps psychosocial support of patients diagnosed with breastcancer; reducing anxiety and depression.. Psychiatr Hung, 28, 454-63 (in Hungarian).

Larkey LK, Roe DJ, Weihs KL, et al (2014). Randomized controlled trial of qigong/tai chi easy on cancer-related fatigue in breast cancer survivors. Ann Behav Med (Epub ahead of print).

Li SX, Liu BB, Lu JH (2014). Longitudinal study of cancerrelated fatigue in patients with colorectal cancer. Asian Pac J Cancer Prev, 15, 3029-33.

Minton O, Richardson A, Sharpe M, et al (2008). A systematic review and meta-analysis of the pharmacological treatment of cancer-related fatigue. J Natl Cancer Inst, 100, 1155-66.

Mitchell SA (2010). Cancer-related fatigue: state of the science. $P M R, 2,364-83$.

Montazeri A, Jarvandi S, Haghighat S, et al (2001). Anxiety and depression in breast cancer patients before and after participation in a cancer support group. Patient Educ Couns, 45, 195-8.

Roth AJ, Nelson C, Rosenfeld B, et al (2010). Methylphenidate for fatigue in ambulatory men with prostate cancer. Cancer, 116, 5102-10.

Schleife H, Sachtleben C, Finck Barboza C, et al (2012). Anxiety, depression, and quality of life in German ambulatory breast cancer patients. Breast Cancer, 21, 208-13.

Schmidt ME, Chang-Claude J, Seibold P, et al (2014). Determinants of long-term fatigue in breast cancer survivors: results of a prospective patient cohort study. Psychooncology.

Smets EM, Garssen B, Bonke B, et al (1995). The Multidimensional Fatigue Inventory (MFI) psychometric qualities of an instrument to assess fatigue. J Psychosom Res, 39, 315-25.
Vodermaier A, Millman RD (2011). Accuracy of the hospital anxiety and depression scale as a screening tool in cancer patients: a systematic review and meta-analysis. Support Care Cancer, 19, 1899-908.

Zigmond AS, Snaith RP (1983). The hospital anxiety and depression scale. Acta Psychiatr Scand, 67, 361-70 